\title{
Rising and sinking intruders in dense granular flows
}

\author{
Lu Jing $\odot,{ }^{1}$ Julio M. Ottino $\odot,{ }^{1,2,3}$ Richard M. Lueptow $\odot,{ }^{1,2,3, *}$ and Paul B. Umbanhowar $\oplus^{2}$ \\ ${ }^{1}$ Department of Chemical and Biological Engineering, Northwestern University, Evanston, Illinois 60208, USA \\ ${ }^{2}$ Department of Mechanical Engineering, Northwestern University, Evanston, Illinois 60208, USA \\ ${ }^{3}$ Northwestern Institute on Complex Systems (NICO), Northwestern University, Evanston, Illinois 60208, USA
}

(Received 7 June 2019; revised manuscript received 26 February 2020; accepted 3 June 2020; published 30 June 2020)

\begin{abstract}
We computationally determine the net bed force on single spherical intruder particles in dense granular flows as a function of particle size, particle density, shear rate, overburden pressure, and gravity. A simple buoyancylike scaling law is recovered (analogous to that in fluids), but with a scale factor that depends on the particle size ratio due to discrete contacts. Comparing the bed force with the intruder weight results in predictions of whether an intruder rises or sinks that agree with data from various independent experiments of free surface granular flows.
\end{abstract}

DOI: 10.1103/PhysRevResearch.2.022069

Intruder particles in fluidized or flowing granular beds tend to segregate (rise or sink) due to their size or density difference with the bed particles [1-15]. Segregation in vibrofluidized systems, known as the Brazil nut effect [3], depends on various mechanisms [1-8] including buoyancy. With sufficient fluidization, the buoyancy force on an intruder follows Archimedes' principle [8], thus explaining the phase transition between normal and reverse Brazil nut effects [5]. In contrast to this clear picture, the force driving segregation in dense granular flows remains elusive. While extensive research has focused on segregation of flowing bidisperse mixtures from the continuum perspective [16,17], quantitative studies of the particle-scale segregation force are fewer and more recent. Guillard et al. [12] proposed a virtual springbased force meter in numerical simulations that allows direct measurement of the segregation force in shear flows. They interpreted the force as summed contributions from normal and shear stress gradients based on two-dimensional wall-driven flow simulations. van der Vaart et al. [14] applied a similar approach in three-dimensional chute flows and decomposed the measured force into lift- and buoyancy-like forces. Despite these insights, a generalized characterization of the segregation force is still lacking in either size [11-15] or density [10] segregation, as well as more complicated situations of combined size and density segregation. For example, Félix and Thomas [9] found an interplay between size and density whereby segregation can change direction (rise or sink) more than once as the intruder size increases. The rise-sink transition depends mainly on size and density ratios but is insensitive to flow geometries (chute, heap, and rotating drum)

\footnotetext{
*r-lueptow@northwestern.edu

Published by the American Physical Society under the terms of the Creative Commons Attribution 4.0 International license. Further distribution of this work must maintain attribution to the author(s) and the published article's title, journal citation, and DOI.
}

or flow rates (e.g., the rotation speed of a drum) [9]. None of the recent segregation force laws $[12,14]$ or state-of-the-art segregation theories [16-20] offer a priori prediction of the entire rise-sink transition.

Here we solve the puzzle by providing a general scaling law that allows shear-induced segregation to be viewed as a result of the imbalance between the gravitational force and a size-corrected buoyancy force. This is achieved by exploring a wide range of size and density ratios under controlled pressure and shear rate in a constant-shear-rate system. The scaling law is confirmed in chute flow simulations where shear rate gradients are small and validated by previous canonical surface flow experiments [9].

Methods. We simulate two flow systems using the discrete element method code LIGGGHTS [21]. The first system consists of a constant-shear-rate geometry [Fig. 1(a)] where bed particles of diameter $d$ and density $\rho$ are sheared in a streamwise $(x)$ and spanwise $(y)$ periodic box of length $30 d$, height $30 d$, and width $10 d$ to $30 d$ (varied as needed) in the presence of gravity (typically, $g=9.81 \mathrm{~m} / \mathrm{s}^{2}$ ). The top (a)

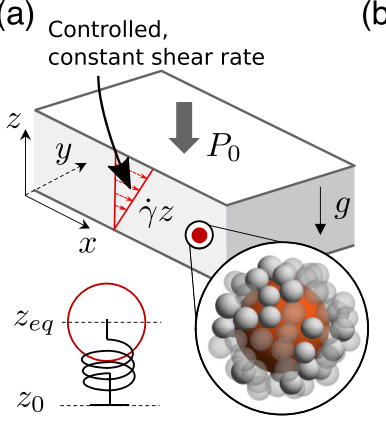

(b)
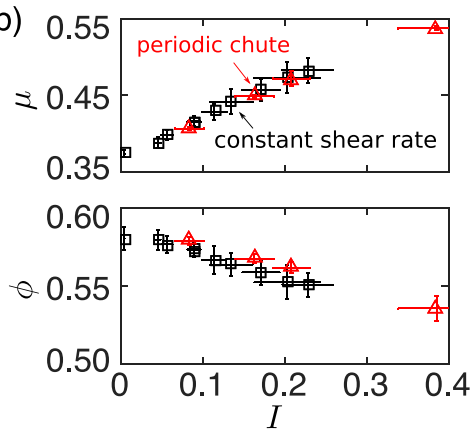

FIG. 1. (a) Intruder particle (red) in a controlled, constant-shearrate flow. A virtual spring measures the net vertical bed force on the intruder. (b) Rheology for constant-shear-rate and chute flows $\left(\theta=\{22,24,25,28\}^{\circ}\right)$. Data are depth-averaged values. Error bars indicate \pm 1 standard deviation. 
and bottom walls are roughened with randomly distributed stationary particles to reduce slip [22], and an overburden pressure $P_{0}$ is applied reactively on the top wall. A constant shear rate $\dot{\gamma}$ is imposed across the geometry by applying a streamwise stabilizing force to each particle (including the intruder); at each time step, for a particle with streamwise velocity $u_{p}$ and vertical position $z_{p}$, a small force proportional to $\dot{\gamma} z_{p}-u_{p}$ is added in the $x$ direction [Fig. 1(a)]. This stabilizing algorithm, commonly used by granular rheologists [23-25] and recently applied to segregation studies [26], can generate constant-shear-rate flows over a wide range of conditions: $600 \mathrm{~Pa} \leqslant P_{0} \leqslant 3000 \mathrm{~Pa}$ and $1 \mathrm{~s}^{-1} \leqslant \dot{\gamma} \leqslant 40 \mathrm{~s}^{-1}$, leading to depth-averaged inertial numbers $I=\dot{\gamma} d \sqrt{\rho / \sigma_{z z}}$ ranging from 0.005 to 0.25 , where $\sigma_{z z}$ is the vertical normal stress. Since we focus on the flow far from rigid boundaries, this geometry can be viewed as a representative volume where the segregation force may be associated with a controlled, locally uniform shear rate and vertical normal stress. The second flow we simulate is inclined chute flow (with slope angle $\theta$ and $g_{z}=g \cos \theta$ ), as used in previous experimental and numerical studies of intruder segregation $[9,14]$. This flow exhibits slightly nonlinear velocity profiles, serving to confirm the generality of the results based on the linearized (but inhomogeneous in stress fields), constant-shear-rate system. The similar values of effective friction $\mu$ and packing fraction $\phi$ in the two systems indicate nearly the same rheology [Fig. 1(b)].

In our simulations, we use bed particles of $d=5 \mathrm{~mm}$ with a $10 \%$ uniform size distribution to avoid layering, $\rho=$ $2500 \mathrm{~kg} / \mathrm{m}^{3}$ (varied to change the density ratio), and material properties similar to glass beads (used in experiments [9]), i.e., the Hertz contact model with Young's modulus $5 \times 10^{7} \mathrm{~Pa}$ (reduced for computational efficiency), Poisson's ratio 0.4, restitution coefficient 0.8 , and friction coefficient 0.5 .

An intruder of diameter $d_{i}$ and density $\rho_{i}$ is placed near the middle of the bed (initial height $z_{0}$ ), with size ratio $R=d_{i} / d$ varying from 0.5 to 8 and density ratio $R_{\rho}=\rho_{i} / \rho$ varying from 0.5 to 3 . To measure the vertical force driving segregation, we follow Guillard et al. [12] and tether the intruder to a vertical spring (leaving free the other five degrees of freedom), which causes it to fluctuate about an equilibrium height $z_{\mathrm{eq}}$ [Fig. 1(a)]. In steady state, the net contact force exerted on the intruder by the neighboring bed particles, the bed force $F$, is balanced by the spring force and the gravitational force, i.e., $F=k\left(z_{\text {eq }}-z_{0}\right)+m_{i} g_{z}$, where $k$ is the virtual spring stiffness and $m_{i}$ is the intruder mass. The spring acts as a force meter and the measurement of $F$ is insensitive to $k[12,14]$. Uncertainties (error bars) of $F$ are estimated considering temporal correlations [27] of the fluctuations of the intruder height about $z_{\mathrm{eq}}$.

Results. Figure 2(a) shows that, for $\rho_{i}=\rho, F$ (symbols) and $m_{i} g_{z}$ (dashed curve) increase similarly with $R$. However, subtle differences between $F$ and $m_{i} g_{z}$ indicate imbalanced forces that drive segregation. To better visualize the differences, the ratio $F / m_{i} g_{z}$ is plotted in Fig. 2(b). Focusing on $R_{\rho}=1, F / m_{i} g_{z}$ is less than one for $R<1$, i.e., a small intruder is pulled down by gravity. As $R$ is increased above one, $F / m_{i} g_{z}$ becomes greater than one, i.e, a large intruder is pushed up by the bed force. These scenarios are consistent with typical percolation and squeeze expulsion explanations for size segregation $[13,28]$. Notably, $F / m_{i} g_{z}$ falls slightly
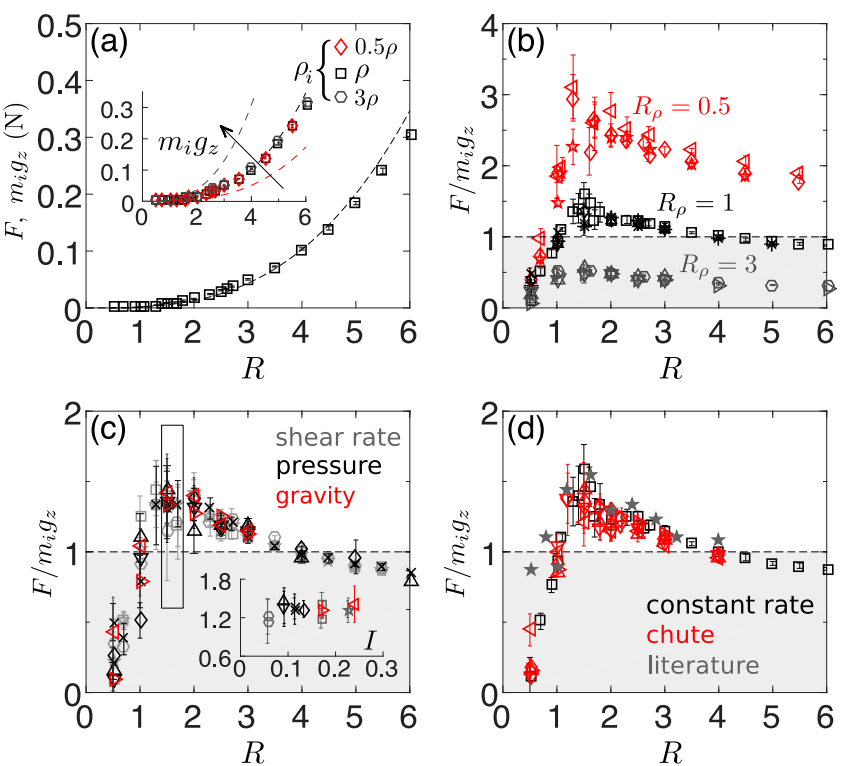

FIG. 2. Dependence of $F$ on various parameters. Data in (a)(c) are from the constant-shear-rate system. (a) $F$ and $m_{i} g_{z}$ vs $R$ for $\rho_{i}=\rho\left(\rho=2500 \mathrm{~kg} / \mathrm{m}^{3}, P_{0}=600 \mathrm{~Pa}, \dot{\gamma}=30 \mathrm{~s}^{-1}, g=9.81 \mathrm{~m} / \mathrm{s}^{2}\right)$. Inset: varying $\rho_{i}$. (b) $F / m_{i} g_{z}$ vs $R$ for $R_{\rho}=\{0.5,1,3\}$ with varying $\rho$ and $\rho_{i}$. (c) $F / m_{i} g_{z}$ vs $R$ for $600 \mathrm{~Pa} \leqslant P_{0} \leqslant 3000 \mathrm{~Pa}, 10 \mathrm{~s}^{-1} \leqslant$ $\dot{\gamma} \leqslant 40 \mathrm{~s}^{-1}$, and $g=\{5,15\} \mathrm{m} / \mathrm{s}^{2}$. Inset: $F / m_{i} g_{z}$ vs $I$ for $1.4 \leqslant R \leqslant$ 1.6 (selected for illustration). (d) Constant-shear-rate results $\left(P_{0}=\right.$ $\left.600 \mathrm{~Pa}, \dot{\gamma}=30 \mathrm{~s}^{-1}\right)$, chute flow results $\left(\theta=\{22,24,25,28\}^{\circ}\right)$, and data from [14]. Shaded (unshaded) areas in (b)-(d) indicate that the intruder sinks (rises) from its initial position.

below one for $R>4$, since $m_{i} g_{z}$ increases more rapidly with $R$ than $F$; thus very large intruders sink. This is consistent with reverse segregation [9].

Next, we vary $R_{\rho}$ by changing $\rho_{i}$. The inset of Fig. 2(a) shows that $F$ remains unchanged as $\rho_{i}$ increases from $0.5 \rho$ to $3 \rho$ (symbols), whereas $m_{i} g_{z}$ obviously depends on $\rho_{i}$ (dashed curves). Therefore, the intruder density does not affect the bed force but alters segregation by changing the ratio $F / m_{i} g_{z}$. Figure 2(b) shows that data for varying $\rho_{i}$ and $\rho$ collapse on curves identified only by $R_{\rho}$, i.e., whether an intruder rises or sinks is determined only by the relative diameter $(R)$ and density $\left(R_{\rho}\right)$.

Figure 2(c) shows that flow conditions $P_{0}, \dot{\gamma}$, and $g$ have no significant impact on $F / m_{i} g_{z}$ over a wide range of variation. As illustrated in Fig. 2(c) inset, $F / m_{i} g_{z}$ is essentially independent of $I$ for $0.05<I<0.25$, a range encompassing typical inertial flows [29]. Reducing $I$ toward the quasistatic limit (typically $10^{-3}$ ) may enhance the segregation force [12], a point we address below.

Finally, Fig. 2(d) shows that results from the constantshear-rate system described to this point are consistent with results from chute flows, including previous simulations of glass beads using a different (linear) contact model [14]; the chute flow results are insensitive to $\theta$. This agreement indicates that $F$ is system invariant for similar granular materials.

Scaling. We now focus on the scaling of $F$ and test a buoyancy-like Archimedes force scale, $\phi \rho g_{z} V_{i}$, where $V_{i}$ is the intruder volume, viewing the granular flow as a "fluid" 

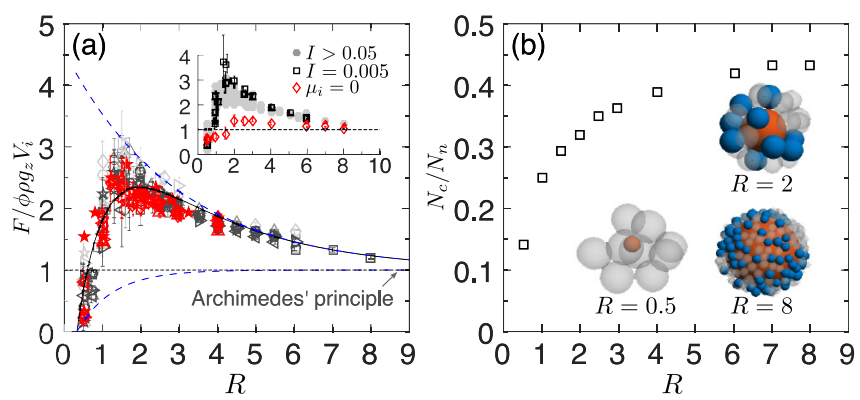

FIG. 3. (a) $F / \phi \rho g_{z} V_{i}$ vs $R$, with varying $\rho_{i}, \rho, P_{0}, \dot{\gamma}, \theta$, and $g$ in constant-shear-rate and chute flows. Data from [14] is also included (red stars). Solid curve is a fit to $F=f(R) \phi \rho g_{z} V_{i}$, with dashed curves showing the two exponential terms defining $f(R)$; see text. Inset: extreme cases with $I=0.005\left(P_{0}=3000 \mathrm{~Pa}, \dot{\gamma}=1 \mathrm{~s}^{-1}\right)$ and $\mu_{i}=0\left(P_{0}=1800 \mathrm{~Pa}, \dot{\gamma}=20 \mathrm{~s}^{-1}\right)$, respectively. (b) $N_{c} / N_{n}$ vs $R$ $\left(P_{0}=2400 \mathrm{~Pa}, \dot{\gamma}=20 \mathrm{~s}^{-1}\right)$. Inset: $x z$-plane view of contact network at various $R$, showing intruders (red), contacting particles (blue), and noncontacting neighbor particles (gray).

of bulk density $\phi \rho$ with normal stress gradient $\phi \rho g_{z}$. Unlike van der Vaart et al. [14], who split $F$ into buoyancy- and lift-like forces, we propose instead that $F$, as a whole, scales like a buoyancy force. This is confirmed in Fig. 3(a), which shows $F / \phi \rho g_{z} V_{i}$ vs $R$ for 252 distinct simulations with all data collapsing onto a master curve. This scaling provides a simple and robust description of the bed force over widely varying flow conditions and is an apparent consequence of force fluctuations, which depend primarily on pressure (gradients) but weakly on flow conditions in inertial granular flows [30].

The master curve in Fig. 3(a) deviates notably from the Archimedean force $F / \phi \rho g_{z} V_{i}=1$, starting below one for $R<$ 1 , increasing toward a maximum at $R \approx 2$, and asymptotically approaching one as $R$ is further increased. The deviation appears to originate in discrete contacts between finite-size particles. A small intruder $(R<1)$ can percolate through voids [13], thereby reducing $F$, i.e., $F / \phi \rho g_{z} V_{i}<1$, while a large intruder $(R>1)$ that preferentially receives large force fluctuations [31] can experience an enhanced $F$, i.e., $F / \phi \rho g_{z} V_{i}>1$. As $R$ further increases $(R \gg 1)$, finite-size effects diminish and the continuum limit $F / \phi \rho g_{z} V_{i}=1$ is recovered. These arguments are qualitatively illustrated in the inset of Fig. 3(b), where distinct contact networks (with blue contacting and gray noncontacting particles around red intruders) are displayed at various $R$. Quantitatively, the relative number of contacts can be characterized by the time-averaged number ratio of contacting neighbor particles $\left(N_{c}\right)$ to all "nearby" particles $\left(N_{n}\right)$, defined as having their centers within $d+d_{i} / 2$ of the intruder center. The number ratio increases with $R$, asymptotically approaching a maximum [Fig. 3(b)], which is consistent with the asymptotic approach of the data in Fig. 3(a) to the limit of Archimedes' buoyancy.

The geometric effects (i.e., non-Archimedian) are associated with the frictional nature of granular contacts, which enhances segregation; this enhancement saturates for $\mu_{i} \gtrsim$ 0.4 [13], where $\mu_{i}$ is the intruder friction coefficient. For frictionless intruders $\left[\mu_{i}=0\right.$ in Fig. 3(a) inset], $F / \phi \rho g_{z} V_{i}$ collapses toward one (closer to Archimedian and hence does not overcome the intruder weight for $R_{\rho}=1$ ), which explains previous observations that large frictionless intruders tend to sink $[13,14]$. In nearly quasistatic flows $[I=0.005$ in Fig. 3(a) inset], $F / \phi \rho g_{z} V_{i}$ is higher, likely due to enhanced frictional resistance to deformation near yielding [32]. This effect tends to plateau above yielding, explaining the insensitivity of $F / m_{i} g_{z}$ to $I$ in Fig. 2(c) inset. A similar trend of enhanced segregation forces at very low $I$ was found in previous twodimensional simulations [12].

Rise-sink transition. It is convenient to express $F$ as $F=$ $f(R) \phi \rho g_{z} V_{i}$, where $f(R)$ is a dimensionless scale factor. It is unclear how to derive $f(R)$ from first principles; extending kinetic theory-based segregation models toward the denseflow limit could be feasible [18,19] but is beyond the scope of this work.

Here we instead choose an empirical expression based on the observation that two geometric effects dominate in different ranges of $R$, i.e., percolation-induced bed force weakening for $R<1$ and particle contact induced force strengthening for $R>1: f(R)=\left(1-c_{1} e^{-R / R_{1}}\right)\left(1+c_{2} e^{-R / R_{2}}\right)$, where $c_{1}$, $c_{2}, R_{1}$, and $R_{2}$ are fitting parameters. The first term [lower dashed curve in Fig. 3(a)] represents stronger percolation (thus smaller bed force) as $R$ decreases; its exponential form is chosen to reconcile the exponential dependency of percolation probability [28] and percolation velocity [33] on $R$. The second term [upper dashed curve in Fig. 3(a)], which decreases toward one as $R$ increases, accounts for the decreased relative number of contacts around the intruder at small $R$ [Fig. 3(b)] and the requirement that the curve asymptotically approaches Archimedes' buoyancy for large $R$. Fitting to the data in Fig. 3(a) gives $c_{1}=1.43, c_{2}=3.55, R_{1}=0.92$, and $R_{2}=$ 2.94, where $R_{1}$ and $R_{2}$ are characteristic size ratios for the two effects to dominate. Interestingly, the fitting parameters are insensitive to shear conditions and material properties (except very low friction; see Supplemental Material [34]). The two terms together recover the continuum argument, $f(\infty) \rightarrow 1$, and the force balance in monodisperse flows, $f(1)=1 / \phi$ (i.e., $F=\rho g_{z} V_{i}$ at $R=1$ ). Although $\phi$ is case specific, the fitting results in $\phi=1 / f(1)=0.55$, a value agreeing with Fig. 1(b), further supporting the model.

The empirical double-exponential model requires only four parameters to describe the nonmonotonic curve, clearly indicates two geometric effects associated with physically reasonable characteristic size ratios, and is appropriately constrained by limiting cases. It provides a simple means to predict segregation based only on $R$ and $R_{\rho}$. An intruder in a flowing bed is "neutral" when the bed force $f(R) \phi \rho g_{z} V_{i}$ offsets its weight $\rho_{i} g V_{i}$, i.e., $R_{\rho}=\phi f(R)$, which describes a curve dividing the $R-R_{\rho}$ space into "rise" (below the curve) and "sink" (above the curve) zones; see Fig. 4. To validate this phase diagram, we first simulate single untethered intruders with varying $d_{i}$ and $\rho_{i}$ in the constant-shear-rate flow, observing whether they rise, sink, or neither (i.e., mean displacement $<3 d$ ) over $500 \mathrm{~s}$ of simulation. The independent simulation results are in excellent agreement with the predictions derived with tethered intruders [Fig. 4(a)].

To further demonstrate the generality of the predicted risesink transition, we compare it with experiments by Félix and Thomas [9], who studied segregation of tracer particles of different sizes and densities in various configurations: 


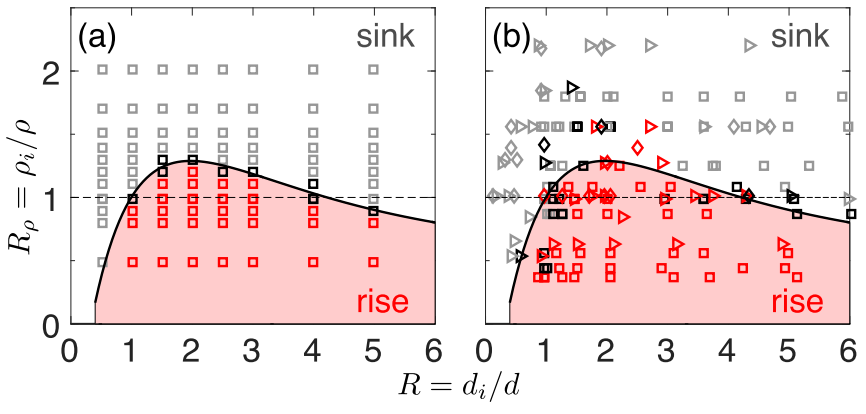

FIG. 4. Predicted rise-sink transition compared to untethered intruders in (a) 88 constant-shear-rate simulations $\left(P_{0}=600 \mathrm{~Pa}\right.$, $\dot{\gamma}=20 \mathrm{~s}^{-1}$ ) and (b) 189 experiments [9]. Curves show $R_{\rho}=\phi f(R)$ with $\phi=0.55$. Red, gray, and black symbols indicate rising, sinking, and neutral intruders, respectively. Data in (b) are from rotating drums $(\square)$, chute flows $(\diamond)$, and heap flows $(\triangleright)$.

rotating drums, chute flows, and heap flows. The experimental results agree well with the predictions of our phase diagram [Fig. 4(b)]. The few mismatches near $R \approx 2$ are expected as experimental results are sensitive near rise-sink transition zones and depend slightly on the detailed criterion for determining the segregation direction [9]. Nevertheless, such complete and accurate prediction of the rise-sink transition is remarkable given the wide range of approaches (simulations and experiments), flow geometries, flow conditions, and particle sizes and densities included in Fig. 4. The agreement demonstrates that the empirical scaling law captures the primary feature of the bed force (i.e., a size-corrected buoyancy force), while other factors (e.g., material properties, geometries, and flow conditions) are secondary, at least for the constant-shear-rate flows and free surface flows we consider.

Discussion. The scaling law we propose for the segregation force has a simple, buoyancy-like form, which is not unfamiliar given that Archimedes' principle with corrections has been applied to creeping granular fluids [35], vibrofluidized granular gases [7,8], and yielding granular solids [32]. The general applicability of this scaling is evident in that a single set of parameters obtained from independent spring-based force measurements predicts rise-sink transitions in a broad range of physical situations involving dense granular flows [9].

This work paves the way for further advancing the understanding of granular segregation. Although our results indicate that $F$ is invariant with varying shear profiles in surface flows, segregation in wall-driven flows (or close to boundaries) exhibits additional dependence on gradients of shear [12], granular temperature [36], and packing density [36,37]. In particular, the shear contribution in $F$ found by Guillard et al. [12] may be attributed to the use of wall-driven flows that exhibit strong shear variations over a distance comparable to the intruder size. This contrasts with surface flows where the velocity profile is nearly linear in the vicinity of an intruder, corresponding to an essentially uniform local shear rate, as is the case here. Nonlocal (or higher-order) effects, particularly those related to shear rate gradients [12], warrant further investigation. Furthermore, the finding that $F / \phi \rho g_{z} V_{i}$ is insensitive to external flow conditions is not to be confused with the known effects of shear rate and confining pressure on segregation velocity $[26,38-40]$. While the direction of segregation is determined by competition between the bed force and the gravitational force, the segregation velocity depends further on resistive forces (often viewed as drag). Understanding the drag force has proved challenging due to the difficulty in isolating driving and drag terms from contact forces $[10,15,41]$. Now, with the generalized driving force model we provide, it is possible to calculate the drag force on moving intruders. It is also relevant to consider varying the particle species concentration around the tethered intruder to include general industrial and geophysical settings [42-47] where the segregation force depends on the particle species concentration $[11,48]$.

Acknowledgments. The authors acknowledge A. Shankar for his contributions to preliminary stages of this work. This research was supported in part through the computational resources and staff contributions provided for the Quest high performance computing facility at Northwestern University which is jointly supported by the Office of the Provost, the Office for Research, and Northwestern University Information Technology.
[1] J. B. Knight, H. M. Jaeger, and S. R. Nagel, Vibration-Induced Size Separation in Granular Media: The Convection Connection, Phys. Rev. Lett. 70, 3728 (1993).

[2] T. Shinbrot and F. J. Muzzio, Reverse Buoyancy in Shaken Granular Beds, Phys. Rev. Lett. 81, 4365 (1998).

[3] M. E. Möbius, B. E. Lauderdale, S. R. Nagel, and H. M. Jaeger, Brazil-nut effect: Size separation of granular particles, Nature (London) 414, 270 (2001).

[4] N. Shishodia and C. R. Wassgren, Particle Segregation in Vibrofluidized Beds Due to Buoyant Forces, Phys. Rev. Lett. 87, 084302 (2001).

[5] D. C. Hong, P. V. Quinn, and S. Luding, Reverse Brazil Nut Problem: Competition Between Percolation and Condensation, Phys. Rev. Lett. 86, 3423 (2001).

[6] A. P. J. Breu, H.-M. Ensner, C. A. Kruelle, and I. Rehberg, Reversing the Brazil-Nut Effect: Competition Between Percolation and Condensation, Phys. Rev. Lett. 90, 014302 (2003).
[7] D. A. Huerta and J. C. Ruiz-Suárez, Vibration-Induced Granular Segregation: A Phenomenon Driven by Three Mechanisms, Phys. Rev. Lett. 92, 114301 (2004).

[8] D. A. Huerta, V. Sosa, M. C. Vargas, and J. C. Ruiz-Suárez, Archimedes' principle in fluidized granular systems, Phys. Rev. E 72, 031307 (2005).

[9] G. Félix and N. Thomas, Evidence of two effects in the size segregation process in dry granular media, Phys. Rev. E 70, 051307 (2004).

[10] A. Tripathi and D. V. Khakhar, Numerical Simulation of the Sedimentation of a Sphere in a Sheared Granular Fluid: A Granular Stokes Experiment, Phys. Rev. Lett. 107, 108001 (2011).

[11] K. van der Vaart, P. Gajjar, G. Epely-Chauvin, N. Andreini, J. M. N. T. Gray, and C. Ancey, Underlying Asymmetry Within Particle Size Segregation, Phys. Rev. Lett. 114, 238001 (2015). 
[12] F. Guillard, Y. Forterre, and O. Pouliquen, Scaling laws for segregation forces in dense sheared granular flows, J. Fluid Mech. 807, R1 (2016).

[13] L. Jing, C. Y. Kwok, and Y. F. Leung, Micromechanical Origin of Particle Size Segregation, Phys. Rev. Lett. 118, 118001 (2017).

[14] K. van der Vaart, M. P. van Schrojenstein Lantman, T. Weinhart, S. Luding, C. Ancey, and A. R. Thornton, Segregation of large particles in dense granular flows suggests a granular Saffman effect, Phys. Rev. Fluids 3, 074303 (2018).

[15] L. Staron, Rising dynamics and lift effect in dense segregating granular flows, Phys. Fluids 30, 123303 (2018).

[16] J. M. N. T. Gray, Particle segregation in dense granular flows, Annu. Rev. Fluid Mech. 50, 407 (2017).

[17] P. B. Umbanhowar, R. M. Lueptow, and J. M. Ottino, Modeling segregation in granular flows, Annu. Rev. Chem. Biomol. Eng. 10, 129 (2019).

[18] J. T. Jenkins and D. K. Yoon, Segregation in Binary Mixtures Under Gravity, Phys. Rev. Lett. 88, 194301 (2002).

[19] L. Trujillo, M. Alam, and H. J. Herrmann, Segregation in a fluidized binary granular mixture: Competition between buoyancy and geometric forces, Europhys. Lett. 64, 190 (2003).

[20] M. Larcher and J. T. Jenkins, The evolution of segregation in dense inclined flows of binary mixtures of spheres, J. Fluid Mech. 782, 405 (2015).

[21] C. Kloss, C. Goniva, A. Hager, S. Amberger, and S. Pirker, Models, algorithms and validation for opensource DEM and CFD-DEM, Prog. Comput. Fluid Dyn. 12, 140 (2012).

[22] L. Jing, C. Y. Kwok, Y. F. Leung, and Y. D. Sobral, Characterization of base roughness for granular chute flows, Phys. Rev. E 94, 052901 (2016).

[23] E. Lerner, G. Düring, and M. Wyart, A unified framework for non-Brownian suspension flows and soft amorphous solids, Proc. Natl. Acad. Sci. USA 109, 4798 (2012).

[24] A. H. Clark, J. D. Thompson, M. D. Shattuck, N. T. Ouellette, and C. S. O'Hern, Critical scaling near the yielding transition in granular media, Phys. Rev. E 97, 062901 (2018).

[25] K. Saitoh and B. P. Tighe, Nonlocal Effects in Inhomogeneous Flows of Soft Athermal Disks, Phys. Rev. Lett. 122, 188001 (2019).

[26] A. M. Fry, P. B. Umbanhowar, J. M. Ottino, and R. M. Lueptow, Effect of pressure on segregation in granular shear flows, Phys. Rev. E 97, 062906 (2018).

[27] N. F. Zhang, Calculation of the uncertainty of the mean of autocorrelated measurements, Metrologia 43, S276 (2006).

[28] S. B. Savage and C. K. K. Lun, Particle size segregation in inclined chute flow of dry cohesionless granular solids, J. Fluid Mech. 189, 311 (1988).

[29] E. Azéma and F. Radjaï, Internal Structure of Inertial Granular Flows, Phys. Rev. Lett. 112, 078001 (2014).

[30] E. Longhi, N. Easwar, and N. Menon, Large Force Fluctuations in a Flowing Granular Medium, Phys. Rev. Lett. 89, 045501 (2002).
[31] C. Voivret, F. Radjaï, J.-Y. Delenne, and M. S. El Youssoufi, Multiscale Force Networks in Highly Polydisperse Granular Media, Phys. Rev. Lett. 102, 178001 (2009).

[32] W. Kang, Y. Feng, C. Liu, and R. Blumenfeld, Archimedes law explains penetration of solids into granular media, Nat. Commun. 9, 1101 (2018).

[33] N. Khola and C. Wassgren, Correlations for shear-induced percolation segregation in granular shear flows, Powder Technol. 288, 441 (2016).

[34] See Supplemental Material at http://link.aps.org/supplemental/ 10.1103/PhysRevResearch.2.022069 for the influence of material properties on the scaling curve.

[35] K. Nichol, A. Zanin, R. Bastien, E. Wandersman, and M. van Hecke, Flow-Induced Agitations Create a Granular Fluid, Phys. Rev. Lett. 104, 078302 (2010).

[36] Y. Fan and K. M. Hill, Phase Transitions in Shear-Induced Segregation of Granular Materials, Phys. Rev. Lett. 106, 218301 (2011).

[37] K. M. Hill and Y. Fan, Isolating Segregation Mechanisms in a Split-Bottom Cell, Phys. Rev. Lett. 101, 088001 (2008).

[38] Y. Fan, C. P. Schlick, P. B. Umbanhowar, J. M. Ottino, and R. M. Lueptow, Modelling size segregation of granular materials: The roles of segregation, advection and diffusion, J. Fluid Mech. 741, 252 (2014).

[39] C. P. Schlick, Y. Fan, A. B. Isner, P. B. Umbanhowar, J. M. Ottino, and R. M. Lueptow, Modeling segregation of bidisperse granular materials using physical control parameters in the quasi-2d bounded heap, AIChE J. 61, 1524 (2015).

[40] S. Liu and J. J. McCarthy, Transport analogy for segregation and granular rheology, Phys. Rev. E 96, 020901(R) (2017).

[41] Y. Duan, P. B. Umbanhowar, J. M. Ottino, and R. M. Lueptow, Segregation models for density-bidisperse granular flows, Phys. Rev. Fluids 5, 044301 (2020).

[42] J. M. N. T. Gray and A. R. Thornton, A theory for particle size segregation in shallow granular free-surface flows, Proc. R. Soc. A 461, 1447 (2005).

[43] D. R. Tunuguntla, O. Bokhove, and A. R. Thornton, A mixture theory for size and density segregation in shallow granular freesurface flows, J. Fluid Mech. 749, 99 (2014).

[44] K. M. Hill and D. S. Tan, Segregation in dense sheared flows: gravity, temperature gradients, and stress partitioning, J. Fluid Mech. 756, 54 (2014).

[45] J. M. N. T. Gray and C. Ancey, Particle-size and -density segregation in granular free-surface flows, J. Fluid Mech. 779, $622(2015)$

[46] H. Xiao, P. B. Umbanhowar, J. M. Ottino, and R. M. Lueptow, Modelling density segregation in flowing bidisperse granular materials, Proc. R. Soc. A 472, 20150856 (2016).

[47] Z. Deng, P. B. Umbanhowar, J. M. Ottino, and R. M. Lueptow, Continuum modelling of segregating tridisperse granular chute flow, Proc. R. Soc. A 474, 20170384 (2018).

[48] R. P. Jones, A. B. Isner, H. Xiao, J. M. Ottino, P. B Umbanhowar, and R. M. Lueptow, Asymmetric concentration dependence of segregation fluxes in granular flows, Phys. Rev. Fluids 3, 094304 (2018) 